\title{
Encapsulation of Menthol in Beeswax by a Supercritical Fluid Technique
}

\author{
Linjing Zhu, ${ }^{1}$ Hongqiao Lan, ${ }^{2}$ Bingjing He, ${ }^{1}$ Wei Hong, ${ }^{1}$ and Jun $\mathrm{Li}^{1}$ \\ ${ }^{1}$ Department of Chemical and Biochemical Engineering, College of Chemistry and Chemical Engineering, \\ Xiamen University, Xiamen 361005, China \\ ${ }^{2}$ Technology Center, China Tobacco Fujian Industry Corporation, Xiamen 361022, China
}

Correspondence should be addressed to Hongqiao Lan, lanhongqiao@fjtic.cn and Jun Li, junnyxm@xmu.edu.cn

Received 1 September 2010; Revised 28 October 2010; Accepted 16 November 2010

Academic Editor: Cordelia Selomulya

Copyright () 2010 Linjing Zhu et al. This is an open access article distributed under the Creative Commons Attribution License, which permits unrestricted use, distribution, and reproduction in any medium, provided the original work is properly cited.

\begin{abstract}
Encapsulation of menthol in beeswax was prepared by a modified particles from gas-saturated solutions (PGSS) process with controlling the gas-saturated solution flow rate. Menthol/beeswax particles with size in the range of $2-50 \mu \mathrm{m}$ were produced. The effects of the process conditions, namely, the pre-expansion pressure, pre-expansion temperature, gas-saturated solution flow rate, and menthol composition, on the particle size, particle size distribution, and menthol encapsulation rate were investigated. Results indicated that in the range of studied conditions, increase of the pressure, decrease of the gas-saturated solution flow rate, and decrease of the menthol mass fraction can decrease the particle size and narrow particle size distribution of the produced menthol/beeswax microparticles. An $\mathrm{N}_{2}$-blowing method was proposed to measure the menthol release from the menthol/beeswax microparticles. Results showed that the microparticles have obvious protection of menthol from its volatilization loss.
\end{abstract}

\section{Introduction}

Natural menthol exists in peppermint and other mint oils, having minty odor. It is widely used as tobacco flavor to manufacture menthol cigarettes, inhibit stimulation from tobacco, and cover miscellaneous gases. However, the melting temperature of menthol is $42 \sim 43^{\circ} \mathrm{C}$ at atmospheric pressure, which makes it easy to sublimate. The high volatility not only means its short storage time and its pollution to the work environment and product line in the menthol cigarette production process but also suggests the flavor's rapid loss with smoking. Obviously, the control and detection of menthol transfer is an important issue during the production, storage, and smoking of menthol cigarette. The encapsulation of menthol will not only provide coolness and refreshing taste from menthol but will also ensure controlled release of menthol; this may also improve the effectiveness, broaden the time range of menthol flavor, and ensure optimal dosage [1]. Hee [2] studied the transfer of menthol from tobacco to filter and the menthol percentages of different menthol cigarettes, indicating that adding menthol to the plate of paper instead of tobacco is the most effective method.
Peng [3] studied the menthol composite particles by using phase separation-condensation method, and results showed that the composite particles with an encapsulation efficiency of menthol up to $58.7 \%$ could not only enhance the retention of menthol in the cigarette but also improve the smoke quality.

In this study, the used wall material is beeswax, which has special fragrance of honey and flour. It can melt at $62 \sim 67^{\circ} \mathrm{C}$ at atmospheric pressure. Furthermore, beeswax completely turns to liquid in the condition of $15 \mathrm{MPa}$ and $55.2^{\circ} \mathrm{C}$ as tested by using laboratory equipment [4].

Conventional methods for preparing composite particles such as spray drying, spray cooling, spray chilling, air-suspension coating, extrusion, centrifugal extrusion, freeze drying, coacervation, rotational suspension separation, cocrystallization, and interfacial polymerization often involve the use of organic solvents, thermal and chemical degradation of compounds, high-energy requirement, multistage processes, environmental pollution, low encapsulation efficiency, and broad particle size distribution. These limitations are the main reasons for the increasing interest in effective and clean encapsulation methods [5]. Particles from 
gas-saturated solutions (PGSS) process proposed by Weidner et al. [6] employed supercritical fluid technology to prepare microparticles. Currently, various studies have focused on the composite particles prepared by using this technique. For example, Rodrigues et al. [7] prepared microcomposites theophylline/hydrogenated palm oil from a conventional PGSS process and found that limited theophylline was encapsulated in hydrogenated palm oil. Wang et al. [8] investigated lipid/ibuprofen system and controlled release is obviously achieved by the process. Vezzù et al. [9] with a PGSS studied the encapsulation of magnetite nanoparticles (MNPs) into triestearin and phosphatidylcholine mixtures to increase their biocompatibility for future applications in the fields of biomedical diagnostics and therapeutic medications. Recently, Pemsel et al. [10] studied the encapsulation of the biopesticide Cydia pomonella granulovirus by PGSS, and the wall materials were an organic matrix together with admixed UV protectants and phagostimulants. Due to the low operation temperature in the process, in particular, the low temperature at the tip of the nozzle exit, PGSS favors food industry, for example, for preventing flavors from volatilizing. In addition, the technique does not need to use water or organic solvents.

Due to the high volatility of menthol, this research will use a modified PGSS process which can control the flow rate of the gas-saturated solution to prepare the menthol/beeswax particles aimed at preventing menthol from volatilization loss. The influences of the pre-expansion pressure, preexpansion temperature, gas-saturated solution flow rate and menthol composition, on the particle size, particle size distribution, and menthol encapsulation efficiency will be investigated. Also, a $\mathrm{N}_{2}$-blowing method will be used to test the menthol release of the microparticles.

\section{Experimental}

2.1. Materials. Menthol (CP, purity $\geqq 99 \%$ ) and beeswax (mixture) were purchased from Xiamen Glass Instrument Co., Ltd. Greenery Reagent. $\mathrm{CO}_{2}$ was provided by Tongan Air Separation \& Special Gas Factory, Xiamen, China. Anhydrous ethanol (analytical grade) and Ether (analytical grade) were provided from the first Chemical Plant of Shanghai Zhenxing. Acetophenone (analytical grade) was provided from Xiamen Glass Instrument Co., Ltd. Greenery Reagent.

2.2. Apparatus and Procedure. The modified PGSS process used in this experiment is shown in Figure 1. As it shows, the major difference of the process from conventional PGSS process is the addition of the two high-pressure pumps (E1 and E2) for delivering gas-saturated solution. The advantage of the delivery of the gas-saturated solution instead of charge of melted solute solution (unsaturated solution before mixing with high pressure $\mathrm{CO}_{2}$ ) is evident: (1) the process can be operated at relatively low temperature due to the depression of the melting temperature of solid solute in high pressure of $\mathrm{CO}_{2}$; (2) the block problem in the nozzle in conventional PGSS process can be effectively reduced with controlling the flow rate of the gas-saturated solution and the corresponding coaxial nozzle designed (a pipe with O.D. of $3 \mathrm{~mm}$ in a pipe with I.D. of $4 \mathrm{~mm}$; the gas-saturated solution passes through the inner pipe and $\mathrm{CO}_{2}$ goes through the gap between the inner and outer pipes); (3) the solute can be mixed with $\mathrm{CO}_{2}$ efficiently by using $\mathrm{E} 2$ to form gas-saturated solution.

The procedure of the process is described as follows.

(1) $\mathrm{CO}_{2}$ is compressed into the gas damper to the desired pre-expansion pressure and enters the thermostatic system (the operating temperature is controlled by the air bath with a precision of $\pm 0.1^{\circ} \mathrm{C}$ ). The compressed $\mathrm{CO}_{2}$ is divided into two parts: one passes through the preheater $\mathrm{C}$ and valve 4 (which was closed before preparing particles), then enters directly into the external tube of the nozzle system; another passes through valve 1 into the high-pressure mixing vessel and mixes with solid materials added before the experiment to form gas-saturated solution.

(2) The gas-saturated solution is pumped by E2 into the vessel for cycling and mixing with a relatively large flow rate (such as more than $5 \sim 10 \mathrm{~mL} / \mathrm{min}$ ) in the case of fast mixing.

(3) After mixing, E1 (E1 and E2 can be combined together if only one pump is available) works after opening valve 3 with a flow rate of $0.1 \sim 1 \mathrm{~mL} / \mathrm{min}$ (other pumps can be chosen for larger or smaller flow rates) to charge the gas-saturated solution into the inner tube of the nozzle system. This gas-saturated solution is atomized by the $\mathrm{CO}_{2}$ from the external tube through a disc $\mathrm{F}$ with a laser-drilled orifice of $80 \mu \mathrm{m}$ into the collector $\mathrm{G}$ to form fine particles. The overall flow rate of $\mathrm{CO}_{2}$ exhausted is measured by a gas flow meter after filtration. All the particles in the collector were taken out after the experiment and stored in a sealed bottle; the encapsulation efficiency of the sample (arbitrarily sampled from the collected particles) was measured right after the collection for a correct indication of the content of menthol on the surface of the particles.

With the use of menthol as the core material and beeswax as the wall material, the above process was implemented. According to the melting point tested for beeswax (the melting point of the mixture is lower than that of beeswax or menthol), the operating temperature is fixed at $60^{\circ} \mathrm{C}$. The orifice diameter of $80 \mu \mathrm{m}$ was selected for convenient control of the process (larger-size nozzle can cause difficulty to maintain a high pressure). The influences of the operating pressure $P_{0}$, flow rate $L$ and menthol mass fraction $C_{0}$ on the formation of the menthol/beeswax particles were investigated; the experiment was arranged as Table 1 shows. Note that the last column provides the measured menthol mass fraction $(C)$ in the produced menthol/beeswax particles, which includes the menthol on the particles surface and in the particles. Table 1 shows that $C$ is very close to $C_{0}$ with a minimum deviation of the two mass fractions of $0.0 \%$ and a maximum deviation of $2.4 \%$. 


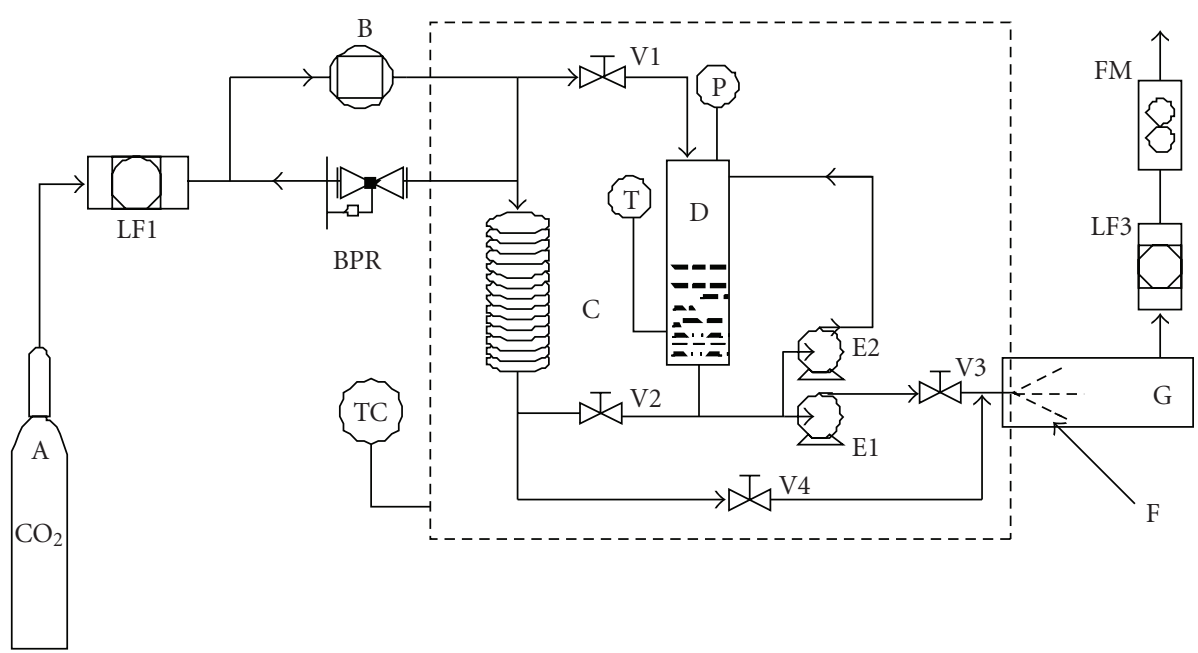

FIGURE 1: Schematic diagram of the modified PGSS process. A: $\mathrm{CO}_{2}$ cylinder; B: compressor; C: preheater; D: mixer; E\#: high-pressure pump; F: double-pass nozzle; G: precipitant; P: pressure gauge; T: thermometer; V\#: valve; FM: flow meter; TC: temperature controller; LF\#: filter; BPR: back pressure valve.

TABle 1: Experiment conditions.

\begin{tabular}{lcccc}
\hline Run no. & $P_{0}, \mathrm{MPa}$ & $L, \mathrm{~mL} / \mathrm{min}$ & $C_{0}$ & $C$ \\
\hline 1 & 6 & 0.21 & $10 \%$ & $10.5 \%$ \\
2 & 10 & 0.21 & $10 \%$ & $9.8 \%$ \\
3 & 15 & 0.21 & $10 \%$ & $10.6 \%$ \\
4 & 20 & 0.21 & $10 \%$ & $11.7 \%$ \\
5 & 15 & 0.21 & $20 \%$ & $20.0 \%$ \\
6 & 15 & 0.21 & $30 \%$ & $32.1 \%$ \\
7 & 15 & 0.21 & $40 \%$ & $38.4 \%$ \\
8 & 15 & 0.11 & $10 \%$ & $12.4 \%$ \\
9 & 15 & 0.40 & $10 \%$ & $11.2 \%$ \\
10 & 15 & 0.81 & $10 \%$ & $12.1 \%$ \\
\hline
\end{tabular}

$P_{0}$ : opterating pressure; $T_{0}$ : operating temperature $=60^{\circ} \mathrm{C} ; \mathrm{D}$ : nozzle size $=80 \mu \mathrm{m} ; \mathrm{L}$ : the flow rate of solution (calibrated with pure water at high pressure); $C_{0}$ : mass fraction of menthol in the menthol/beeswax mixture; $C$ : measured menthol mass fraction in the produced menthol/beeswax particles.

2.3. Analysis Methods. The morphology and size of the menthol/beeswax particles were analyzed by XL30 environment scanning electron microscope (SEM). The particle size and size distribution were analyzed by a laser particle size analyzer (LS908, OMEC, China) with static method.

The menthol encapsulation efficiency in the menthol/ beeswax particles is calculated by

encapsulation efficiency (\%)

$$
=\frac{\text { total menthol }(\mathrm{g})-\text { menthol on thesurface }(\mathrm{g})}{\text { total menthol }(\mathrm{g})} \times 100 \text {, }
$$

where the menthol content on the surface of the particles and the total menthol content were measured by gas chromatography (GC) (FID as the detector; SE-54 column: $30 \mathrm{~m} \times 0.32 \mathrm{~mm} \times 0.25 \mu \mathrm{m}$; nitrogen as the carrier gas with a flow rate of $1 \mathrm{~mL} / \mathrm{min}$; column temperature: $70^{\circ} \mathrm{C}$ for $1 \mathrm{~min}$, then $5^{\circ} \mathrm{C} / \mathrm{min}$, and finally $140^{\circ} \mathrm{C}$ for $1 \mathrm{~min}$; injection port temperature $250^{\circ}$; detector temperature $250^{\circ} \mathrm{C}$; split ratio $50: 1$; injection volume $0.4 \mu \mathrm{L}$ ). Ethanol was used to dissolve the menthol on the surface of the produced particles and ether was used to dissolve the total menthol of the particles.

During the storage of the menthol/beeswax particles, the menthol in the particles will escape via volatilization because of the influence of the external environment. So, the release of the core material is an important indication for evaluating the composite particles, which is calculated by

$$
\begin{aligned}
& \text { menthol release }(\%) \\
& =\frac{\text { menthol content }(\mathrm{g}) \text { at time } t}{\text { initial menthol content }(\mathrm{g})} \times 100,
\end{aligned}
$$

where the initial content of menthol measured by GC is indicated in Table 1. The menthol content at time $t$ is measured by a $\mathrm{N}_{2}$-blowing method: the menthol/beeswax particles sealed in a U-tube, $\mathrm{N}_{2}$ with a pressure of $0.15 \mathrm{MPa}$ passes through the sample in the glass tube; the U-tube is weighed after an interval time to obtain the released amount of menthol in the particles.

\section{Results and Discussion}

3.1. The influence of Pre-Expansion Pressure. The effects of four pre-expansion pressures $(6,10,15$, and $20 \mathrm{MPa})$ were investigated on the formed menthol/beeswax particles when other conditions were fixed $\left(C_{0}=10 \% ; L=0.21 \mathrm{~mL} / \mathrm{min}\right.$; $\left.T_{0}=60^{\circ} \mathrm{C} ; D=80 \mu \mathrm{m}\right)$. Figure 2 gives the average particle size, particle size distribution (PSD), and the encapsulation efficiency of the produced particles. Because the produced particles have very similar morphology, Figure $2(\mathrm{~d})$ provides only a typical SEM image for the menthol/beeswax particles produced at $10 \mathrm{MPa}$ (because of the low melting temperature of the particles, SEM with small scale cannot be performed). 


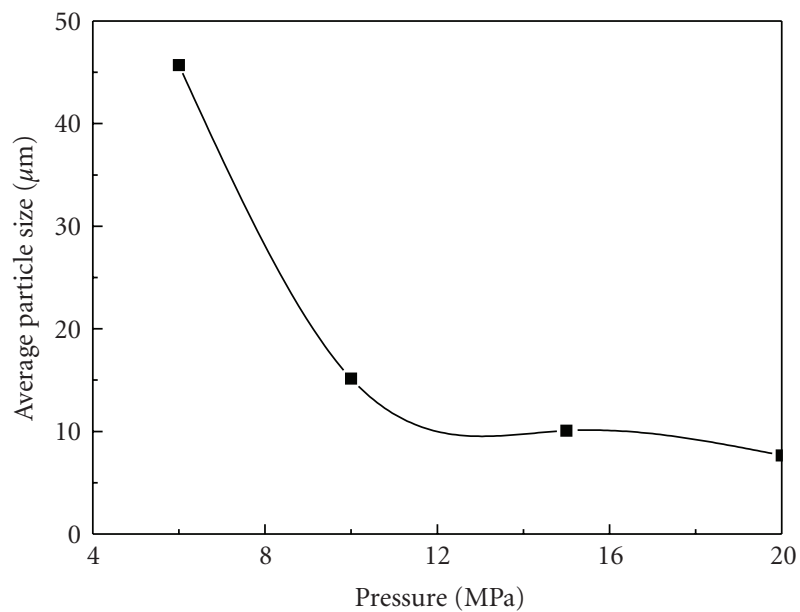

(a)

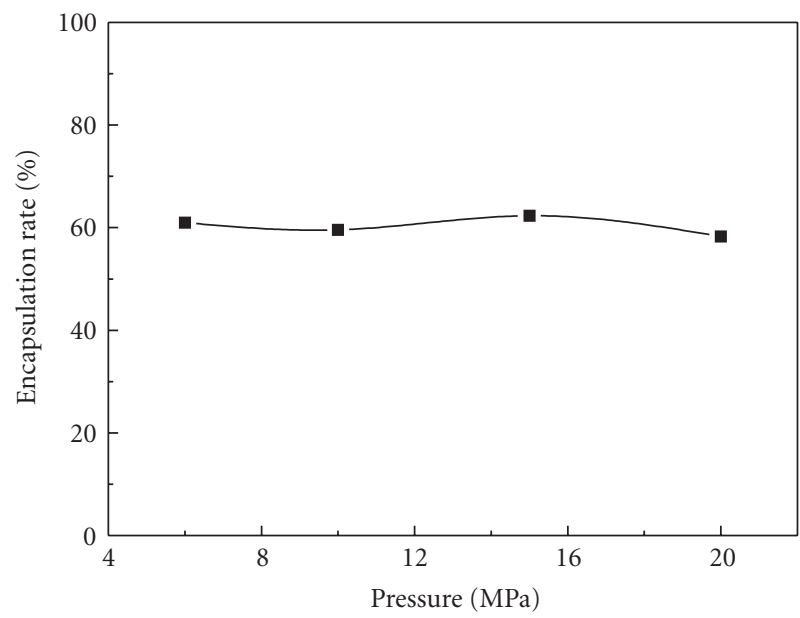

(c)

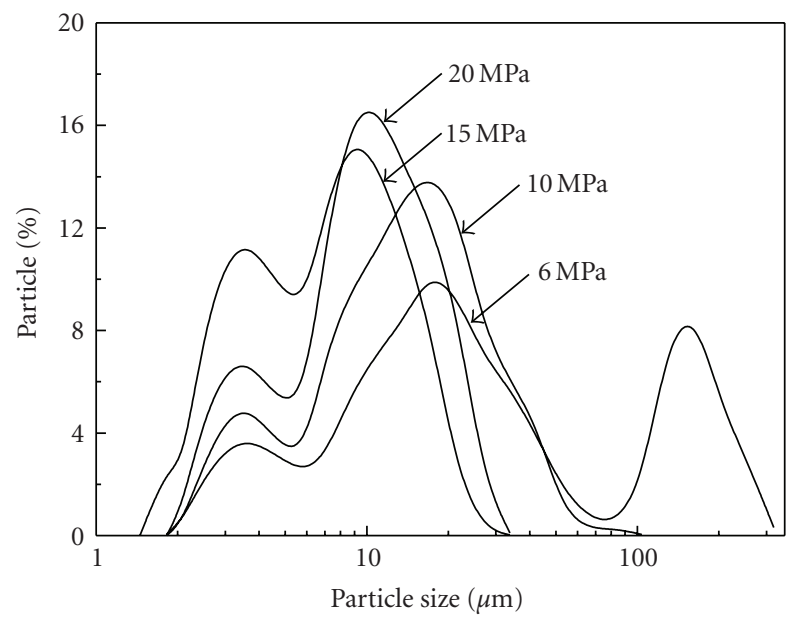

(b)

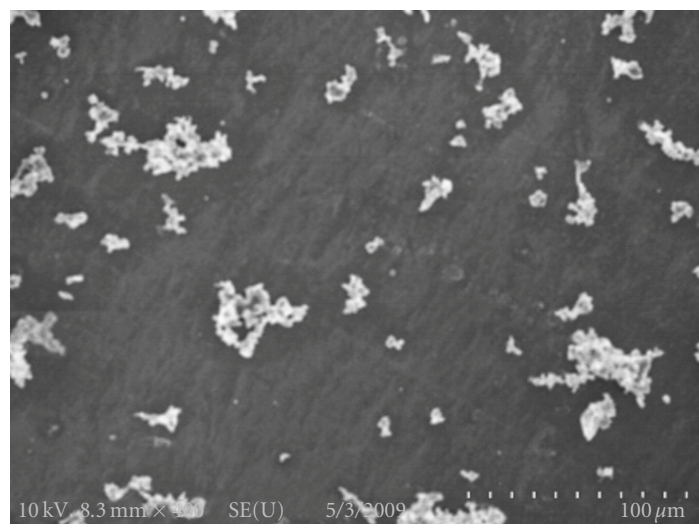

(d)

FIGURE 2: Influence of the pre-expansion pressure on (a) average particle size, (b) PSD, (c) encapsulation efficiency, and (d) SEM image of the menthol/beeswax particles produced at $10 \mathrm{MPa}$.

As shows in Figure 2, as the pressure increases, the average particle size decreases, and particle size distribution becomes narrow. The menthol/beeswax particles prepared at $6 \mathrm{MPa}$ were the largest with an average size of $45 \mu \mathrm{m}$ due to an additional peak appearing at $180 \mu \mathrm{m}$. This mainly attributes to particle aggregation, because the temperature drop at the nozzle exit was not enough when low pressure of $\mathrm{CO}_{2}$ is applied, leading to relatively slow solidification of the menthol/beeswax mixture. The menthol/beeswax particles prepared at other pressures have two peaks (bimodal distribution) obviously from different particle formation mechanisms [11]. Lower pressure tends to diminish the first peak corresponding particles considered to be precipitated from $\mathrm{CO}_{2}$ phase, which is in agreement with the previous modeling conclusions [11]. Figure 2(c) shows that the encapsulation efficiency of all the menthol/beeswax particles is about $60 \%$, meaning that the pre-expansion pressure has no effect on the encapsulation efficiency. Since the encapsulation efficiency is approximately $60 \%$ for all particles with different sizes, this result may also suggest that menthol could be dispersed into beeswax in a relative homogeneous way. In addition, the agreement of the measured menthol content via GC, including that on the particles surface and in the particles, in the produced menthol/beeswax particles arbitrarily sampled and the mass fraction of menthol in the initial menthol/beeswax mixture (see the last two columns in Table 1) also supports the relative homogeneity of the produced particles.

3.2. The Influence of the Flow Rate of Solution. As mentioned, the obvious advantage of the modified PGSS process is the control of the gas-saturated solution. Consequently, different solution flow rates $(0.11,0.21,0.40$, and $0.81 \mathrm{~mL} / \mathrm{min})$ were investigated, while other conditions were fixed $\left(C_{0}=10 \%\right.$; $\left.P=15 \mathrm{MPa} ; T_{0}=60^{\circ} \mathrm{C} ; D=80 \mu \mathrm{m}\right)$. Figure 3 provides the average particle size, PSD, and the encapsulation efficiency of the produced menthol/beeswax particles. 


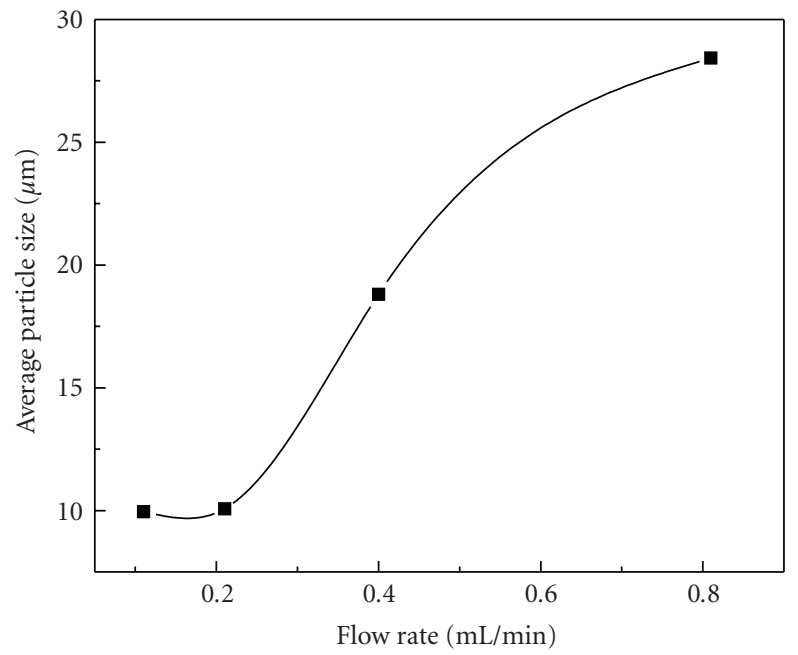

(a)

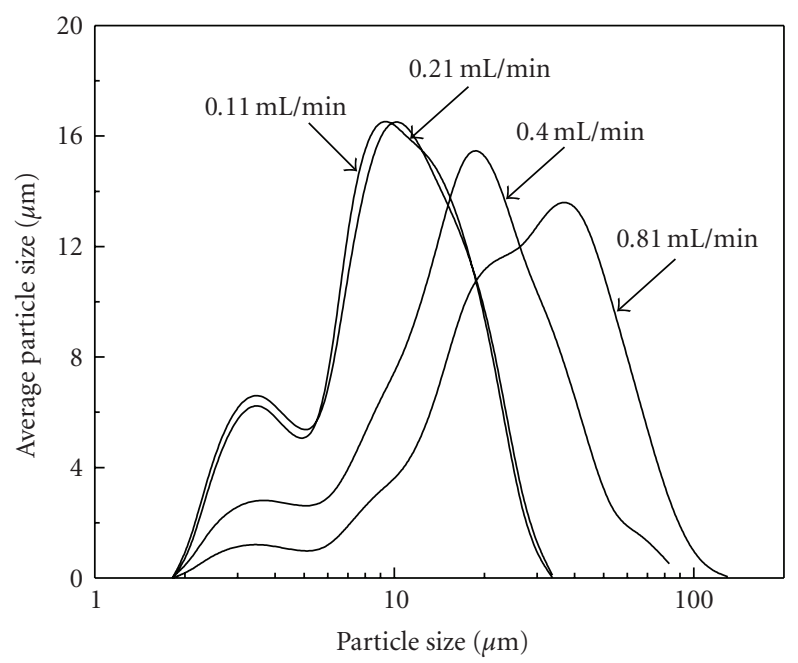

(b)

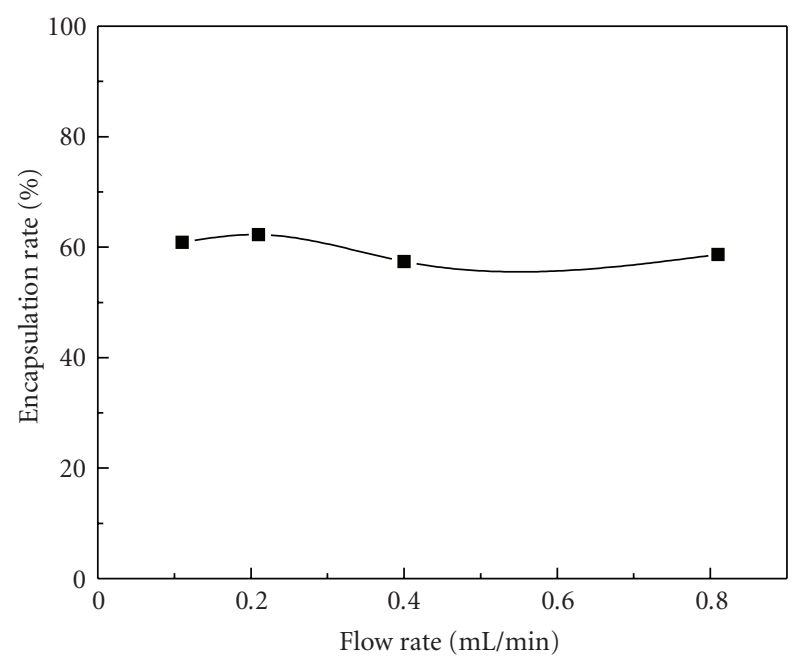

(c)

FIGURE 3: Influence of the flow rate on (a) average particle size, (b) PSD, and (c) encapsulation efficiency.
As shown in Figure 3, the flow rate has an obvious effect on the produced particles: as the flow rate increases, the average particle size increases, and PSD becomes broadened. The menthol/beeswax particles produced at lower flow rate such as 0.11 and $0.21 \mathrm{~mL} / \mathrm{min}$ have very close particle size and similar PSD (bimodal distribution). Yet, the menthol/beeswax particles produced at larger flow rate such as 0.40 and $0.81 \mathrm{~mL} / \mathrm{min}$ tend to diminish the first peak, indicating that atomization mechanism is in dominance at lower solution rate (but this factor was not included in the modeling [11] because the model is only for a semibatch PGSS process). Similarly, Figure 3(c) shows that the encapsulation efficiency of all the menthol/beeswax particles is about $60 \%$, indicating that the flow rate has no obvious effect on the encapsulation efficiency.

3.3. The Influence of the Initial Menthol Content. As the menthol content will affect the encapsulation efficiency, its effect on the formed menthol/beeswax particles was investigated. Four different initial menthol mass fractions, namely, $10 \%, 20 \%, 30 \%$, and $40 \%$, were implemented with fixing the pre-expansion pressure of $15 \mathrm{MPa}$ and the flow rate of $0.11 \mathrm{~mL} / \mathrm{min}$. Figure 4 provides the average particle size, PSD, and the encapsulation efficiency of the produced menthol/beeswax particles.

As shown in Figure 4, as the menthol content increases, the average particle size slightly increases, and the effect disappears when the mass fraction arrives at about $30 \%$. Yet, the effect of the menthol content on the encapsulation efficiency of the composite particles is obvious: higher menthol concentration causes to lower encapsulation efficiency. This is reasonable because higher menthol mass fraction in the initial menthol/beeswax mixture will cause more menthol to stay on the surface of the produced particles.

3.4. Release Curve. For the $\mathrm{N}_{2}$-blowing method, only the menthol/beeswax particles produced at $P_{0}=10 \mathrm{MPa} ; C_{0}=$ $10 \% ; L=0.21 \mathrm{~mL} / \mathrm{min} ; T_{0}=60^{\circ} \mathrm{C} ; D=80 \mu \mathrm{m}$ (see Figure 2(d) for the particles) were tested. A U-tube loaded with $0.1 \mathrm{~g}$ sample and immersed in a water bath at $30^{\circ} \mathrm{C}$ was connected to a $\mathrm{N}_{2}$ cylinder with a reducing valve; the $\mathrm{N}_{2}(0.15 \mathrm{MPa})$ passed through the sample to obtain the retention of menthol in the menthol/beeswax particles at different interval times. Figure 5 gives the menthol release from the particles, and it also provides the menthol release from a physical mixture of menthol/beeswax with $C_{0}=10 \%$ for comparison. The figure shows that after the purge of $\mathrm{N}_{2}$ for about $80 \mathrm{~h}$, the residue of menthol in the menthol/beeswax particles steadily stops at $54 \%$. This residue is consistent with the measured encapsulation rate (59.6\%) indicated in Figure 2(c) for the same sample, indicating that the $\mathrm{N}_{2}$-blowing experiment is also available for measuring the encapsulation efficiency. From the release curve, it clearly shows that the menthol on the surface of the menthol/beeswax particles will gradually run away and cannot be protected for long time. On the other hand, the menthol in the physical mixture loses quickly $(90 \%$ of menthol in the mixture releases in $20 \mathrm{~h}$ ); the slow release 


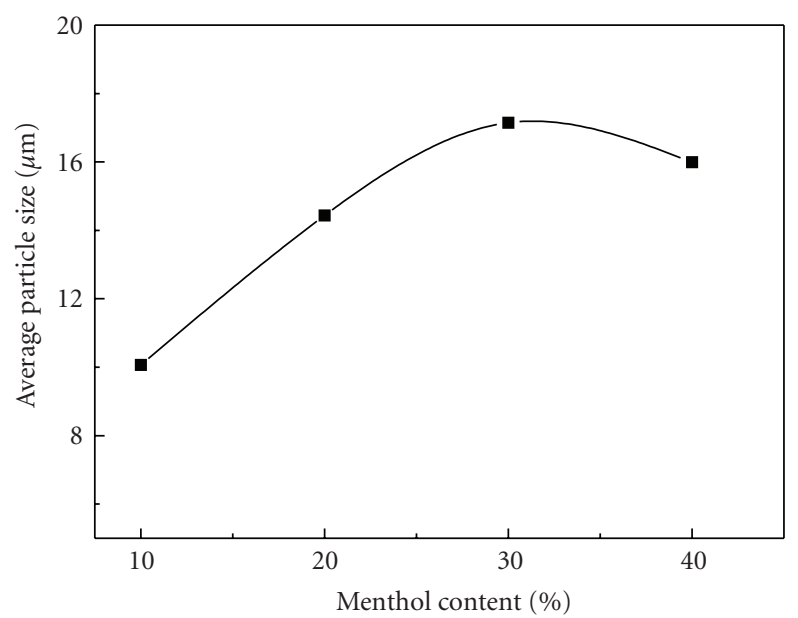

(a)

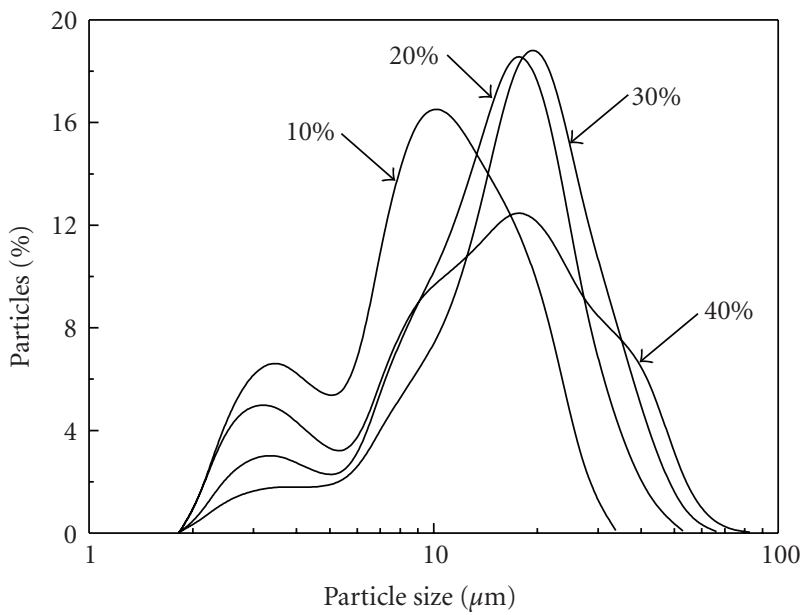

(b)

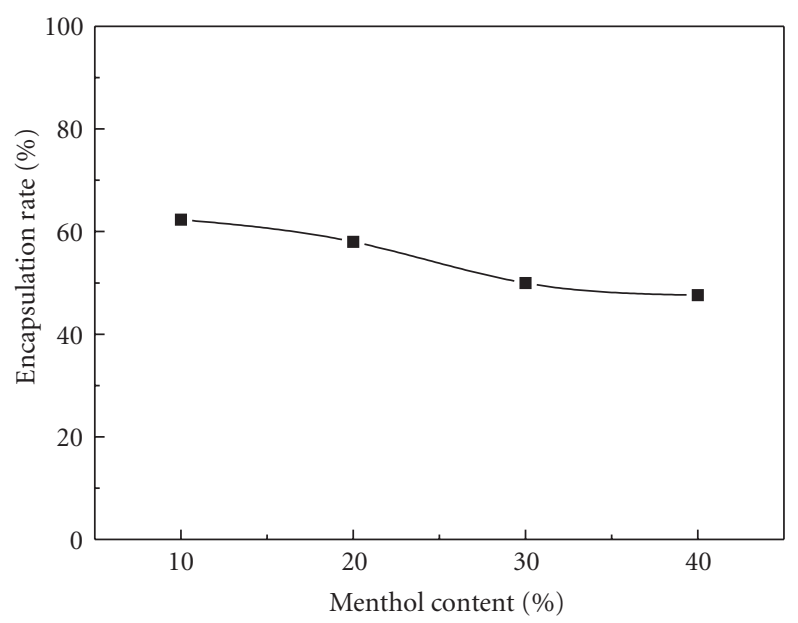

(c)

FIGURE 4: Influence of the initial menthol content on (a) average particle size, (b) PSD, and (c) encapsulation efficiency.

of the left menthol after $20 \mathrm{~h}$ (namely, only about $5 \%$ of menthol releases during 20 to $40 \mathrm{~h}$ ) may be attributed to the adsorption of the menthol on the surface of beeswax particles.

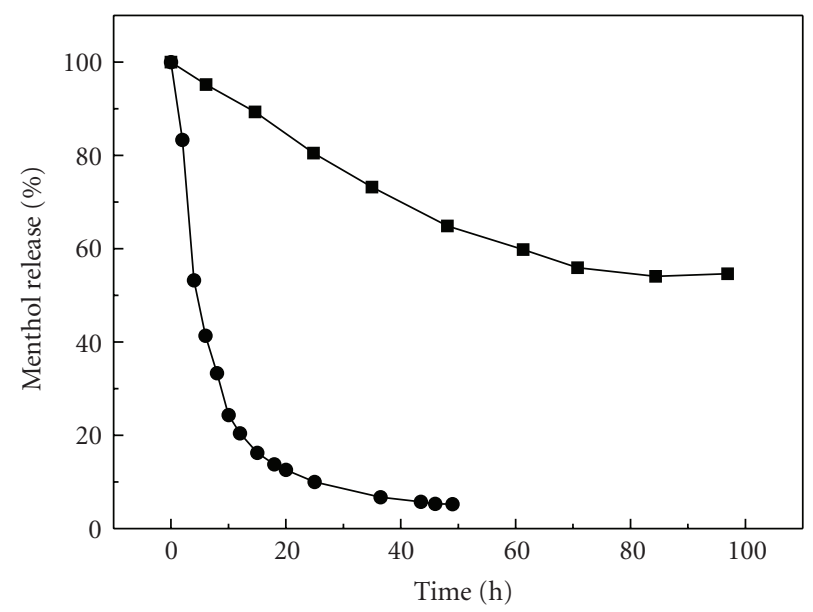

FIGURE 5: Menthol release from the microparticles $(\boldsymbol{\square})$ and physical mixture (0) of menthol/beeswax.

\section{Conclusions}

A modified PGSS process with control of gas-saturated solution flow rate was used to produce menthol/beeswax microparticles in order to protect menthol from volatilizing. The effect of the process parameters, namely, the preexpansion pressure, the gas-saturated solution flow rate, and the menthol mass fraction in menthol/beeswax mixture, on the particle size, particle size distribution, and menthol encapsulation efficiency was investigated. Furthermore, a $\mathrm{N}_{2}$-blowing method was proposed to measure the menthol release from the produced menthol/beeswax microparticles. From these studies, the following conclusions can be drawn.

(1) The modified PGSS process can be employed to prepare menthol/beeswax microparticles with size in the range of $2 \sim 50 \mu \mathrm{m}$. The menthol content measured by GC in the produced particles is consistent with the initial menthol mass fraction in the menthol/beeswax mixture, indicating uniform composite particles were obtained by the process.

(2) Both the pre-expansion pressure and gas-saturated solution flow rate have evident influence on the size of composite particles: as the pre-expansion pressure increases, the gas-saturated solution flow rate decreases, the average size of menthol/beeswax particles decreases and the particle size distribution becomes narrow. Both the pre-expansion pressure and gas-saturated solution flow rate have no obvious influence on the encapsulation efficiency of menthol.

(3) As the menthol mass fraction increases, the average particle size slightly increases, and the encapsulation efficiency of menthol decreases.

(4) The $\mathrm{N}_{2}$-flush measurement for the release of menthol from the menthol/beeswax particles indicates that the microparticles have obvious protection of menthol from its volatilization loss although the menthol on the menthol/beeswax particles surface will volatilize 
gradually. The $\mathrm{N}_{2}$-flush technique is also available for measuring the encapsulation efficiency.

\section{Acknowledgments}

For financial support, the authors are grateful to SRF for ROCS, SEM, NCET of Fujian Province, NSFC, China (Project no. 20876127), and Tobacco S\&T Major Project of China National Tobacco Corporation (Project no. 11020092021).

\section{References}

[1] S. Gouin, "Microencapsulation: industrial appraisal of existing technologies and trends," Trends in Food Science and Technology, vol. 15, no. 7-8, pp. 330-347, 2004.

[2] J. J. Hee, "A study on menthol migration patternsin different mentholated cigarettes," Journal of the Korea Society of Tobacco Science, vol. 23, pp. 77-81, 2001.

[3] R. H. Peng, "Menthol composite particles prepared by phase separation-coacervation method experiment," Tobacco Science, vol. 8, pp. 27-28, 2003.

[4] J. Li, M. Rodrigues, A. Paiva, H. A. Matos, and E. G. De Azevedo, "Binary solid-liquid-gas equilibrium of the tripalmitin $/ \mathrm{CO}_{2}$ and ubiquinone/ $\mathrm{CO}_{2}$ systems," Fluid Phase Equilibria, vol. 241, no. 1-2, pp. 196-204, 2006.

[5] I. Garay, A. Pocheville, and L. Madariaga, "Polymeric microparticles prepared by supercritical antisolvent precipitation," Powder Technology, vol. 197, no. 3, pp. 211-217, 2010.

[6] E. Weidner, R. Steiner, Z. Knez, and Z. Novak, "Powder generation form polyethyleneglycols with compressible fluids," High Pressure Chemical Engineering, vol. 12, pp. 223-228, 1996.

[7] M. Rodrigues, N. Peiriço, H. Matos, E. Gomes De Azevedo, M. R. Lobato, and A. J. Almeida, "Microcomposites theophylline/hydrogenated palm oil from a PGSS process for controlled drug delivery systems," Journal of Supercritical Fluids, vol. 29, no. 1-2, pp. 175-184, 2004.

[8] X. Wang, Y. N. Guo, H. Chen et al., "Composite microparticles of ibuprofen/lipid generated by supercritical fluids from their melts," Frontiers of Chemical Engineering in China, vol. 2, no. 4, pp. 361-367, 2008.

[9] K. Vezzù, C. Campolmi, and A. Bertucco, "Production of lipid microparticles magnetically active by a supercritical fluidbased process," International Journal of Chemical Engineering, vol. 2009, Article ID 781247, 9 pages, 2009.

[10] M. Pemsel, S. Schwab, A. Scheurer, D. Freitag, R. Schatz, and E. Schlücker, "Advanced PGSS process for the encapsulation of the biopesticide Cydia pomonella granulovirus," Journal of Supercritical Fluids, vol. 53, no. 1-3, pp. 174-178, 2010.

[11] J. Li, M. Rodrigues, A. Paiva, H. A. Matos, and E. G. De Azevedo, "Modeling of the PGSS process by crystallization and atomization," AIChE Journal, vol. 51, no. 8, pp. 2343-2357, 2005. 

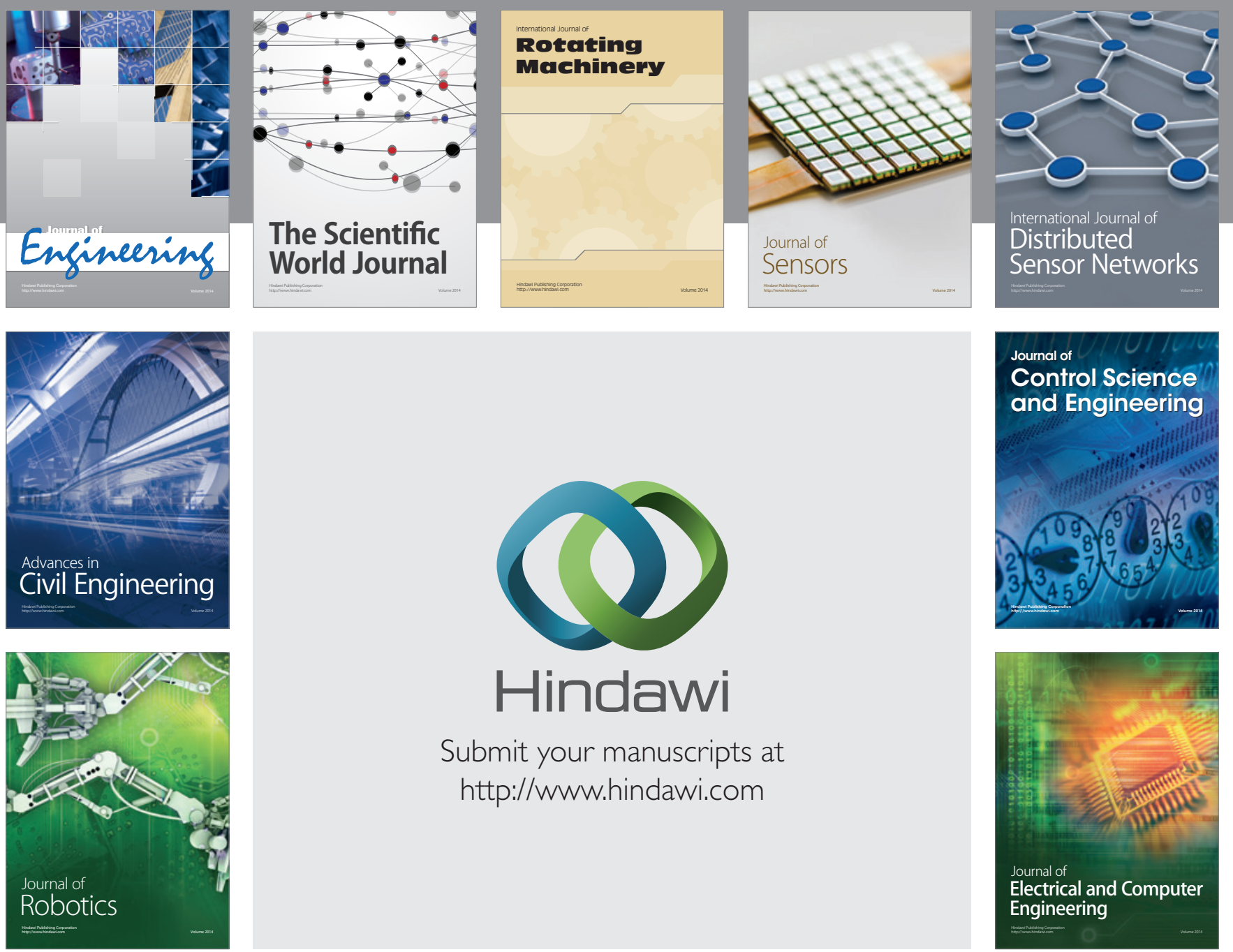

Submit your manuscripts at

http://www.hindawi.com
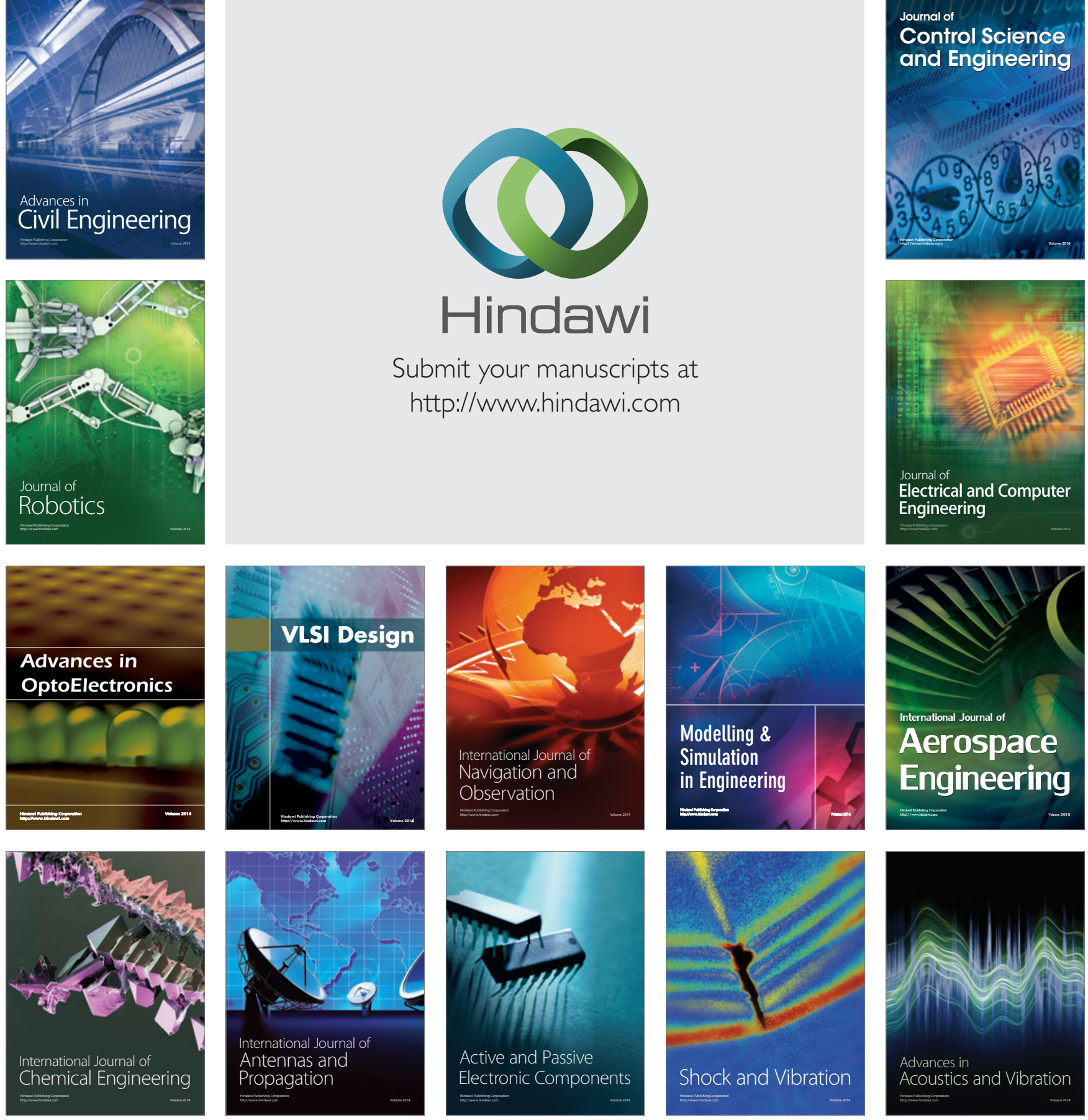